\title{
Surface-enhanced Raman scattering (SERS) of Methyl Orange on Ag-DLC nanoparticles
}

\author{
Arezou Zarei ${ }^{1}$, Azizollah Shafiekhani ${ }^{1,2}$
}

\footnotetext{
${ }^{1}$ Department of Physics, Faculty of Physics and Chemistry, Alzahra University, Vanak, Tehran, Iran..

${ }^{2}$ School of Physics, Institute for Research in Fundamental Sciences, P.O. Box 19395-5531, Tehran, Iran.
}

\begin{abstract}
In the present study, Ag nanoparticles were synthesized in amorphous hydrogenated carbon films on glass substrates by RF-PECVD and RF-sputtering co-deposition method at the room temperature. Methyl orange was utilized as an analyte with different concentrations on $\mathrm{Ag}$ nanoparticles that were embedded in diamond-like carbon (DLC). Ultraviolet-visible (Uv-vis) spectroscopy, XRD analysis, Raman spectroscopy, Atomic Force Microscopy (AFM) and Field Emission Scanning Electron Microscopy (FESEM) were performed to characterize films. Ag-DLC with an average size of less than $14 \mathrm{~nm}$ was the active site for surface-enhanced Raman scattering (SERS). Sensitivity of measurements in SERS spectra was increased by these types of thin films. This method of nano-particle synthesis is cost-effective and just requires a one-step synthesis. Such substrates can be used for several times. Moreover, they are useful for biosensors because of their hardness and other properties that may be subsequently referred.
\end{abstract}

Keywords: Surface-enhanced Raman scattering (SERS), Methyl orange (MO), Diamond-like carbon, Ag nanoparticles. 